\title{
Fair and Popularity based Content Allocation Scheme for IPTV Delivery Networks
}

\author{
Suliman Mohamed Fati \\ Faculty of Information and \\ Communication Technology, \\ UTAR, 31900, Perak Malaysia
}

\author{
Putra Sumari \\ School of Computer Sciences, \\ Universiti Sains Malaysia, \\ 11800, Pinang, Malaysia
}

\author{
Rahmat Budiartu \\ College of Computer Science \\ and Information Technology, \\ Albaha University \\ Albaha, Saudi Arabia
}

\begin{abstract}
IPTV gained a significant attention from researchers and Internet service providers due to its ability to benefit from the capabilities of IP based networks to deliver TV related services with high level of QoS. One of the main problems in IPTV Delivery Networks is how to manage the huge amount of multimedia contents efficiently to meet the demands of users especially for Video on Demand (VoD) services. IPTV Delivery Networks is employed to manage the storage of VoD contents in IPTV system but the dynamic changes in both $\mathrm{VoD}$ contents and users interests make the need for efficient content management is crucial. Many content allocation schemes are proposed for IPTV Delivery Networks to manage the contents efficiently in hierarchical and distributed architectures. Peer-service architecture of IPTV Delivery Networks is efficient and provides high level of QoS but does not take load balance factor of storage servers into consideration. This paper aims to investigate load imbalance problem in IPTV Delivery Networks, modify peer-service architecture, and propose a novel content allocation method that solves the load imbalance in peer-service content network by replicating the contents based on their popularity and the workload of servers within the service area. Experimental results show that this proposed balanced and popularity based content allocation method can maintains the load balancing among servers and avoid the over/under utilization of servers.
\end{abstract}

\section{General Terms}

Multimedia, IPTV, Content Management, Video on Demand.

\section{Keywords}

IPTV, IPTV Delivery Networks, Content Allocation, Peerservice architecture, VOD.

\section{INTRODUCTION}

Internet Protocol Television (IPTV) improves the delivery of TV related services to be transported over IP-based networks to benefit from the high speed of these networks $[1,2]$. Most of the Internet service providers compete to provide IPTV services on their dedicated networks to increase their profits and maximize their base customers [3]. So IPTV services became popular during the last few years since it can deliver high quality viewing service any time[4], and expected to occupy a third of TV viewing markets in 2012 [5]. IPTV can provide Live TV, video on demand, and any additional value added service through the QoS guaranteed IP networks using triple play concept [6]. The main issue that faces the technology is how to efficiently store the huge amount of multimedia data for reusability purpose within the constraints of limited storage and bandwidth capacity to achieve the provider's goals (i.e. maximizing the base customers and profit) and concurrently achieving the customers' goal (i.e. high quality service with cheaper price) [7-9, 34]. So, distributing these multimedia contents over a set of servers among a wide geographical area to satisfy the needs of customers in these areas is considered the optimal solution to reduce the overload on the backbone network and in the same time satisfy the customers' need efficiently. This concept called in the literature, Content Distribution Networks (CDN) [10-13, 35].

However, in a dynamic System like IPTV, the contents are increasing massively, so the management process of these contents surely important topic, and considered the crucial point to achieve a successful IPTV system and still needs more investigation to build efficient and cost effective architecture without violating the load balancing constraints among storage servers. The goal of content management is to deliver these contents efficiently and dynamically among the video servers within IPTV Delivery Networks.

\subsection{Architecture of IPTV Delivery Networks and our proposed Architecture}

In VoD context, many Content storage management architecture models are proposed: single point architecture in which all clients are connected to a single server that stores all the multimedia contents. The main disadvantage of single point architecture is that the load will be focused on a single server and can lead to the server overloading which may cause failure of server (i.e. single point failure). To reduce the load on the main server, many cache servers are allocated within networks to distribute the load among them (distributed model). The hierarchal architecture is proposed to improve the reliability and QoS level but the cost of this architecture is very high. In hierarchical model, the servers are allocated hierarchically like a tree. A novel model called peer service area architecture, in which the IPTV Delivery Networks is divided into many peer-service areas with a cluster of servers for each. The customer has to belong to only one service area and can request the video from any server within his service area. The requested video that does not exist in the service must be redirected to the nearest service area. According to $\mathrm{Li}$ and $\mathrm{Wu}$ in [4], this architecture can satisfy the QoS requirement and also the reliability; and they stated that it is very suitable for IPTV services. However, they focused only on how to produce cost effective IPTV DELIVERY NETWORKS without taking into consideration the load of the servers within service area where they assume that the servers will be classified into two types: Type1 (for high popularity contents) and Type 2 for (low popularity contents) and they assume that $60 \%$ of contents (popular) will be stored in Type 1 servers and low popular (40\% of contents) will be stored in Type 2 servers.

From the viewpoint of load balancing, this architecture has the following limitations (34-35): (1). storing high popular videos in Type 1 servers and low popular videos in Type 2 servers 
may lead to overloading the Type1 servers while Type2 servers will be underutilized which lead to resource wasting of Type 2 that can be used to reduce the load on Type 1 servers efficiently and also this wasting in resources may lead to redundant cost that can be avoided. (2). Replication of popular videos is crucial, and storing the popular video without replication may lead to user request rejection and then degrade the reliability and QoS requirement. (3). the load on each server must be balanced and also the user requests must be distributed on all servers to ensure that no rejection will be occurred for any request.

Based on the aforementioned problems, we propose a modified peer-service area architecture that overcomes those problems in order to build balanced IPTV DELIVERY NETWORKS for IPTV services. In our proposed model, the servers in each service area will be considered the same type and allocated in the same place. The popular video will be replicated based on both of their popularity and the available number of servers to avoid the redundant replication and also avoid the under-replication that may lead to user request rejection. The other feature of our proposed architecture is to add request dispatcher to each service area that controls the distribution of requests for a certain video among the servers that contain a copy of the requested video. Figure. 1 depicts the proposed balanced architecture.

In this paper, the main contribution is demonstrating the load balance of the proposed architecture in figure. 1 by building balanced content allocation scheme to distribute the videos among servers based on their expected load, number of servers, and their maximum load. The rest of this paper is organized as follows: section II gives a comprehensive overview of related works of content allocation problem. Section III exhibits the proposed solution. Section IV analyzes the experimental results. And finally conclusion and future work is provided in Section V.

\section{RELATED WORKS}

Content allocation is considered important point in Content Distribution Network for IPTV technology; many studies are proposed to solve the problem of content allocation. These studies can be classified into central, hierarchical, distributed, and finally peer-service models based on the network architecture that exploited. In central model, the authors allocate the contents into array of disks for single server using striping or replication or both. In [14], the file is divided and then associated with heat ratio to allocate it to disk with lowest heat. Unlike heat based allocation, authors in [15] replicated each stripped block of content randomly to two disks. Tang et al. [16] considered the content allocation to multiple disks as optimization problem to minimize both of storage capacity and waiting time and solve it using Hybrid Genetic Algorithm. Genetic algorithm also incorporated with modified Bin-packing algorithm by [17] to allocate contents with minimum storage capacity and block probability. The trade-off between the storage capacity and concurrent access for each video is discussed in [18] to find the optimal allocation on RAID-3 based on this trade-off.

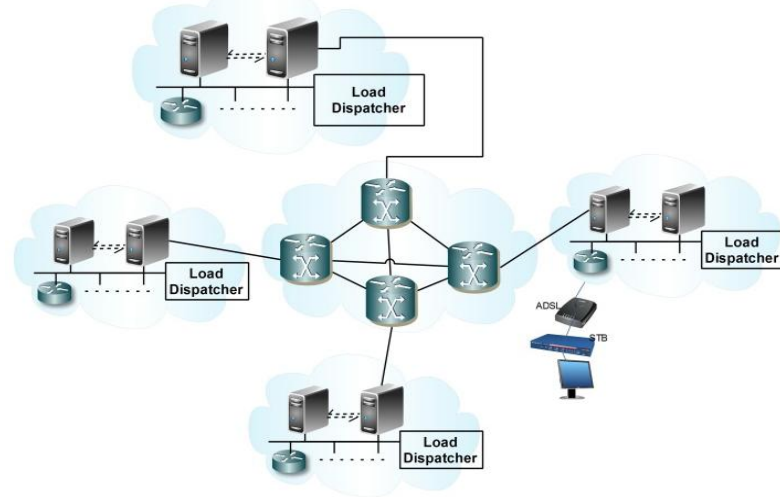

Fig 1: The Proposed Peer-Service Area Architecture For Iptv Delivery Networks

In hierarchical model, the proposed algorithms try to allocate the contents as closer to the users as possible to increase the availability of data and minimize the waiting time of users. In [19] the contents are divided into three classes based on their popularity: 1st class of popularity is stored in Local Service Center (LSC), 2nd class of popularity is stored in Local Central Service Center (LCSC), and 3rd class which will be stored beside copies of members of 1 st and 2 nd classes in Central Service Center (CSC); cost function of capacity and links between the three levels is used to determine number of movies in LSC and LSCS. The videos can be also associated with weights to decide the popular and unpopular movies as discussed in [20] which replicate the popular movies in the leaf cache servers and allocate the unpopular movies in the node 0 (main servers). The threshold value is used in [21] to decide which movies are popular (larger than threshold) and which movies are unpopular (less than threshold) in order to replicate the popular movies in the cache servers and discard the others from replication process. Tsao et al. in [22] took into consideration the connectivity and access probability of each server to produce balanced content allocation, they replicated the high ratio movies to the cache servers with lowest connectivity and access probability based on the determined number of copies. The low ratio videos will be stored in tertiary storage devices. Fetching distance as cost function is used by [23] to optimize the content allocation process. Greedy heuristic algorithm is proposed to allocate content based on cost function. iGreedy algorithm is also proposed to minimize the storage capacity by eliminating the replicas from ancestor nodes if the video already stored in their leaf nodes. Nakaniwa and Ebara in [10] proposed optimal content allocation by maximizing the system reliability as objective function and satisfying time delay as constraint. SMART servers are proposed by [13] to distribute the contents efficiently from global server to local servers. Dynamic Programming is exploited in [24] to allocate contents among the network nodes to minimize the storage and transmission cost of the contents. Authors in [25] replicated the most requested contents into $\mathrm{n} * \mathrm{k}$ nodes where $\mathrm{n}$ : number of nodes and $\mathrm{k}$ : number of servers.

Unlike hierarchical model, in distributed model, the servers are allocated in wide geographic areas without central control; user can access the movie from any site via User Interface Module. The video object can be stripped and distributed and also replicated sequentially into many storage nodes as discussed in [26]. According to [27], the videos are allocated optimally by minimizing the total cost of data transfer using Genetic Algorithm, Mean Field Annealing, and Simulated 
Evolution algorithms. Wang and Guha in [28] proposed two data allocation algorithms: Bandwidth Weighted Partition (BWP) and Popularity Based (PB) algorithms. In both algorithms, the video will be partitioned into unequal chunks and distributed among storage servers according to the bandwidth of servers and popularity of video. No replication process is applied in this study. Authors in [29] tried to minimize the number of traversed hops in the IPTV DELIVERY NETWORKS to deliver contents efficiently using randomization, Popularity, greedy single and greedy global replications. Tsang and Kwok in [30] Proposed a predictive video allocation and replication algorithm based on predictive popularity of videos.

Finally, in peer-service area architecture, the whole area is divided into set of service areas with cluster of servers for each. According to [4], each service area contains type 1 servers to store popular movies and Type 2 servers for storing unpopular movies. $\mathrm{Li}$ and $\mathrm{Wu}$ [4] proposed a content allocation algorithm in which $60 \%$ of the contents are considered high popular and stored in Type1 servers and the rest of content $40 \%$ of movies are stored in Type 2 servers.

Other unclassified works include [1, 11, 31, 32, and 33]: Carnor in [11] proposed a general framework for content allocation called spectrum content management system which consists of three modules: content manager, policy manager, and storage manager. Content migration method called (SXS) is proposed by Ebara [31] which choose the best server to move content to target server with minimum transmission cost. Lee [1] proposed user utility function which reflects the user viewing interests to replicate the movies with high user utility function on the users' devices for IPTV pre-recorded contents. Authors in [32] proposed efficient allocation method to deal with addition, deletion of nodes using tiger hash function and mapping methods. In [33] object placement adjustment with replication is proposed to minimize block probability, in which the object move from high traffic intensity storage server to lower traffic intensity servers.

\section{PROPOSED ALLOCATION SCHEME}

In this proposed solution to solve the problem of load imbalance, balanced content allocation scheme will be built to allocate the contents among the servers within the service area fairly based on the expected load of each video and the maximum capacity of each server. Before demonstrating the proposed solution, we have to explain the used terms and equations.

Let $L_{i}$ denotes the maximum number of requests per unit time [23] that may be serviced by video $i$ and if we assume that each service has its own subscribers who can access the contents that stored in the service area and can be calculated roughly by the subscribers in the whole network divided by the number of service areas. So, the expected load for video $i$ can be computed as the following:

$L_{i}=\left\lceil\frac{U}{S} * \lambda * p_{i j}\right\rceil$

Where $U / S$ represents the number of subscribers who belong to the service area, $\lambda$ represents the request rate per user within unit time, $p_{i j}$ represents the popularity of video $i$ that is stored in server $j$. and the expected load for server $j$ can be expressed by summing the load for all videos that are stored in this server as follows:

$L_{j}=\left\lceil\frac{U}{S} * \lambda * \sum_{i \epsilon j} p i j\right\rceil$
The number of copies that must be allocated for each video must be controlled by the popularity of the video and the available servers. i.e. video $i$ can be allocated on all servers if its popularity is very high to ensure that the expected load can be distributed to minimize the user request rejection. On the other hand, there is no need to replicate the very low popular videos so that one copy is enough to catch the expected load. Based on the illustration above, we can formulate the number of copies for video $i$ as follows:

$C_{i}=\left\lceil S * p_{i}\right\rceil$

Where $C_{i}$ represents the expected number of copies for video $i, S$ represents the number of available servers, and finally, the operator $[$.$] is a ceiling function operator to take the largest$ integer nearest to calculated term.

After computing the expected number of copies for video $i$, we can now calculate the expected load for each copy by dividing the expected load for video $i$ on number of copies as follows:

$L_{c i}=L_{i} / C_{i}$

So, the contents will be allocated based on their popularity such that the low popularity movies will be stored without replication and their load will be computed as in equation (1). Whereas the popular movies will be replicated based on their relative popularity and then their expected load will be distributed on more than one server based on the number of copies as will be explained in the next section.

\subsection{Balanced Content allocation Scheme}

Balanced content allocation scheme aims to place each content to its appropriate server according to its expected load which calculated from equations $(1,3$, and 4$)$ based on their popularity (i.e. the low popular movies will not replicated but the scheme will compute its expected load from equation (1) and then allocated whereas, the popular movies will be replicated and then each replica will be allocated based on their relative expected load as in equation (4). So, firstly the popularity of each movie will be computed based on the Zipf's law as follows [30]:

$p_{i}=\frac{\sum_{j=1}^{M} \frac{1}{j}}{i}$

And then decide the threshold at which the sorted contents list will be divided into two lists: high popular contents list and low popular contents list. This threshold is sensitive and must be deal with it carefully due to the inaccurate adjusting of this threshold may leads to replicating unwanted contents which waste resources or on the other side, un-replicating a popular movies will leads to increasing the load on a certain server and make the system unbalance. So the threshold must be accurately and carefully determined. Finally the low popular movies allocated to servers using Round Robin Algorithm and the high popular movies allocated to servers based on the expected number of replica and the trade-off between the expected load and the maximum server load as depicted in figure.2. 
1. Compute the popularity for each movie $i$.

2. Order the contents based on their popularity in decreasing order.

3. Decide the threshold that classifies the contents into low popular and high popular movies.

4. For low popular movies, apply the Round Robin algorithm to allocate them to servers evenly and compute the current load of the server as: Current_load $+=\mathrm{L}_{\mathrm{i}}$;

5. For high popular movies, do the following for each movie:

1. Compute the number of copies for movie $\mathrm{i}$ based on equation (3).

2. Compute the expected load for these copies based on equation (4).

3. For each copy, find the server with minimum current load and then assign the copy to this server and then update the current load for server as follows:

Current_load $+=\mathrm{L}_{\mathrm{ci}}$

Fig 2: Balanced Content Allocation Scheme

\section{EXPERIMENTAL RESULT}

In this section, we demonstrate the superiority of the proposed content allocation scheme in the modified architecture from the perspective of load balancing on the original scheme in [4]. We implement using $\mathrm{VC}++$ the proposed scheme with that in cost-effective model by $\mathrm{Li}$ and $\mathrm{Wu}$ [4] in order to compare them and show how cost-effective model violates the load balancing condition for peer-service area architecture and also prove that the proposed model with its scheme overcome the problems of their model. The load distribution between servers, threshold value that distinguish the high popular from low popular movies, and also the size of contents in addition to the request rejection rate are examined and analyzed.

There are a set of assumptions that taken into account during the experimental study of the proposed model as following:

$\mathrm{S}=4$ servers, $\mathrm{U}=10000$ users/area. Finally $\lambda=0.01$ request/user/second. We assume that popularity distribution of movies follows the Zipf's law. For the purpose of comparison,

we set the value of threshold to be $60 \%$ according to the

assumption of $\mathrm{Li}$ and $\mathrm{Wu}$. Figure. 3 shows the current load (concurrent accesses) for each server in a single service area; and it is clear that in cost-effective model, server 4 which store $40 \%$ of movies (low popular movies only) has the lowest load while the other servers have higher loads especially server.

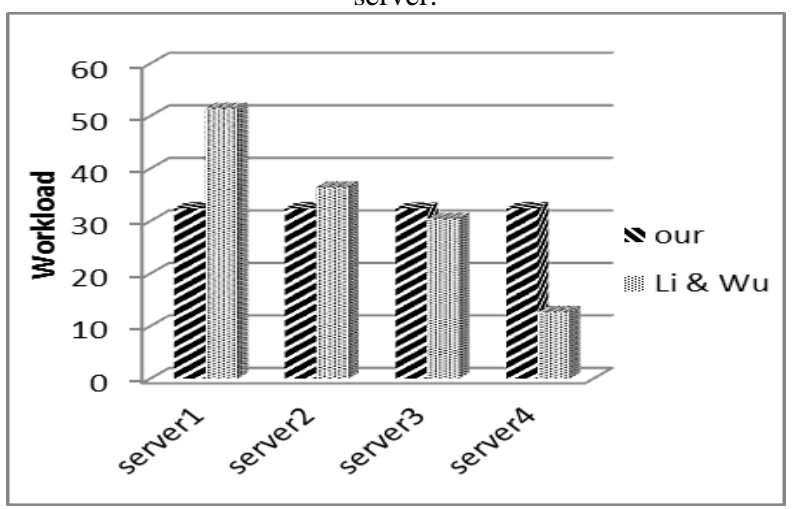

Fig 3: Comparison of Load between the two schemes

This can be interpreted as the following: cost-effective model stores the low popular movies in Type 2 server (server 4 ) and the high popular movies are distributed among the other servers without any consideration to the load of those servers; so this model wastes the resources of some servers without benefit from them that causes over-utilizing the other servers. This scheme may lead to user request rejection at server 1 if it exceeds its maximum load at any time. As depicted in figure.3, the proposed scheme distributes the load among servers evenly due to the proposed scheme allocates the contents according to current load of servers and the maximum load of servers.

\subsection{The effect of threshold}

The threshold values that distinguish the high popular from low popular contents are examined in order to study the effect of threshold change on the load distribution. We fixed the number of contents to be 100 movies and then we carried out both schemes on the following threshold values $10 \%, 30 \%$, $60 \%$, and $90 \%$. Figures. 4 shows the current load of servers in cost-effective model, in this figure we can see clearly that server 4 is affected by threshold value significantly because of the threshold value determine the size of contents that must be stored in server 4 that interpreted as following:

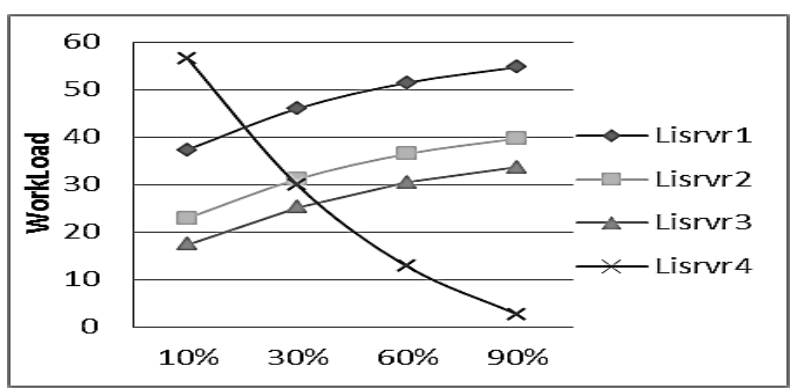

Fig 4: Effect of threshold on cost-eff. scheme

when the threshold value decreases, the contents that will be stored in server 4 increase which leads to increasing the load on server 4 . For example, when threshold $=10 \%$ the load on server4 (Type2 server) is higher than the other servers due to that it stores $90 \%$ of whole movies. As shown in figure 4 also, the other servers suffer from the same problem of load imbalance. In figure.5, we can note that the proposed model isn't affected by the change of this threshold where the proposed model retains its stability and the load of both servers is approximately equal with a slight variation and we think it is negligible. This proves that the proposed model is scalable and efficient under any load variation.

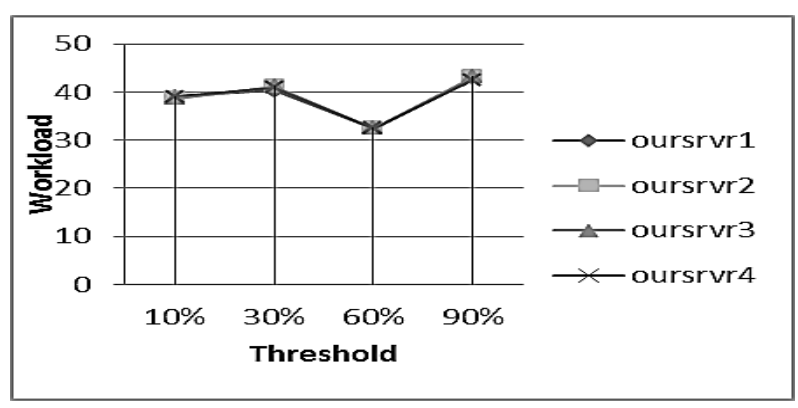

Fig 5: Effect of threshold on the proposed scheme

\subsection{Effects of Content size (number of videos)}

We also tested the effect of the content size on both schemes to check the ability to retain the load balance under any size of contents, Figures. 6 and 7 show the ability of the proposed scheme to retain the load balancing when the content size varying between $100,300,500$, and 700 movies as shown in 
figure.7. On the other hand, the scheme of cost-effective model follows the same behavior of load imbalance among servers under the change in contents sizes as depicted in figure.6.

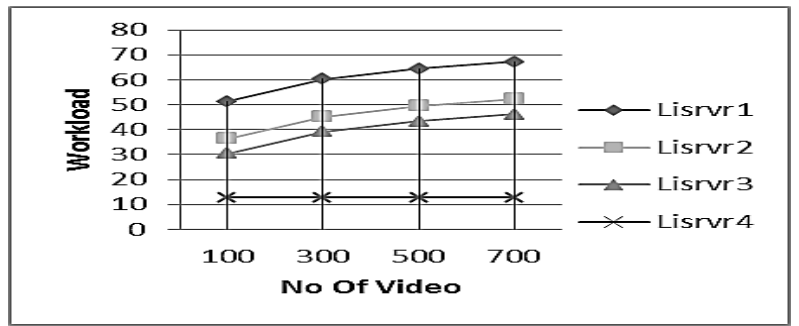

Fig 6: Effect of content size on cost-eff. scheme

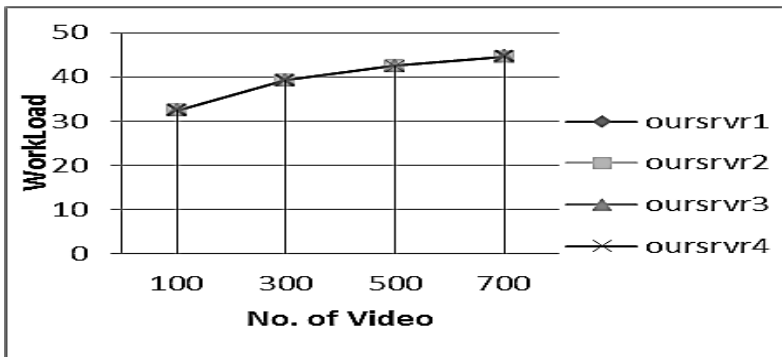

Fig 7: Effect of content size on the proposed scheme

\subsection{Request Rejection Rate}

Request Rejection (RR) Rate can be computed as the ratio between rejected requests and accepted requests, so in this case, when the maximum server load $(\operatorname{Lmax}=50)$ we can prove easily that the content allocation scheme in costeffective model gains high RR rate as depicted in figure8. In the proposed content allocation scheme, the scheme checks firstly the maximum load of server before allocating the movie, so Request Rejection can be avoided or at least minimized significantly. In other words, there is no server overloaded or exceeding the maximum load value in the proposed scheme.

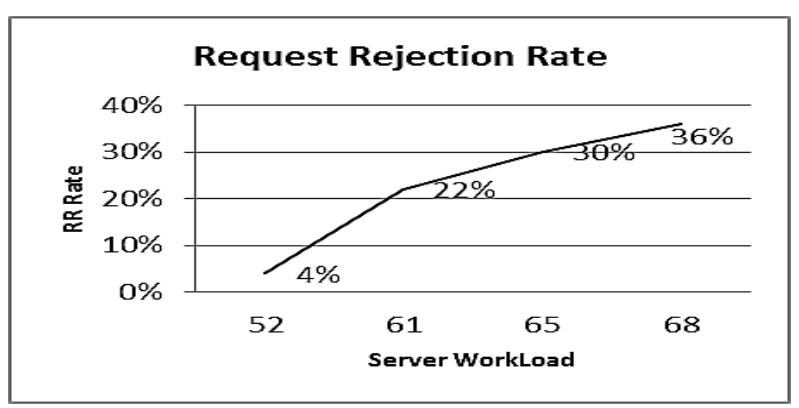

Fig 8: Request Rejection Rate

\section{CONCLUSION AND FUTURE WORK}

This paper studied the load imbalance problem in content allocation process of peer-service architecture of IPTV Delivery Networks for IPTV. In this architecture, the whole area is divided into service areas, and each service area allocates a cluster of servers. The number of requests per unit time is taken as metric of load and we proposed a balanced content allocation scheme that replicates the contents based on the expected load and popularity in addition to the maximum load of servers. The effects of threshold value - that decide the popular and unpopular contents - are studied, in addition to the content size that must be allocated. The experimental results demonstrate the superiority of the proposed scheme on the method of $\mathrm{Li}$ and $\mathrm{Wu}$ [4] from the viewpoint of load balancing.

Many issues still unsolved including the efficient distribution of users' requests between servers, extending the effectivecost of peer-service architecture to include the load balance factor into consideration to optimize the number of servers and allocated contents dynamically to achieve balanced and effective-cost architecture.

\section{REFERENCES}

[1] S. Lee; G. Muntean, A. Smeaton, "Performance-Aware Replication of Distributed Pre-Recorded IPTV Content," IEEE Trans. on Broadcasting, vol.55(2), pp.516-526, June 2009.

[2] S. Mandal, M. MBuru, "Intelligent Pre-fetching toReduce Channel Switching Delay in IPTV Systems," Texas A\&M University, 2008.

[3] A. Yarali, A. Cherry, "Internet Protocol Television (IPTV)," IEEE Region10(TENCON05),pp.1-6,doi: 10.1109/TENCON.2005.300861.

[4] M. Li, C. Wu, "A cost-effective resource allocation and management scheme for content networks supporting IPTV

services," Comput.Commun. 33(1),Jan.2010,doi:10.1016 /j.comcom.2009.08.003.

[5] J. Cheng, "Report: One-third of TV watching to be video-on-demand by2012", Report, 2007.

[6] J. Gu, J. Nah, "Key Management for Overlay-based IPTV Content Delivery", IJCSNS, VOL.8 No.12, Dec. 2008.

[7] P. Pfeffer, "IPTV: Technology and Development Predictions," Fbr \& Integ.Opt.,vol.25,pp325-346,2006, doi:10.1080/01468030600816979.

[8] R. Doverspike, G. Li, K. Oikonomou, K. Ramakrishnan, R. Sinha, D. Wang,C. Chase,"Designing a Reliable IPTV Network," IEEE Internet Comp., vol.13(3),May2009,pp.15-22, DOI $=10.1109 / \mathrm{MIC} .2009 .58$

[9] B. Krogfoss, L. Sofman, A. Agrawal, "Caching architectureand optimization strategies for IPTV networks," Bell Lab Tech. Journal, vol. 13(3), pp. 1328,2008, doi:10.1002/bltj.20320.

[10] A. Nakaniwa, H. Ebara,"Optimal allocation of cache servers and content files in content distribution networks,"Proc. of IASTED Eur. Conf.: internet and multimedia systems and applications (IMSA'07),pp. 1522,2007, Anaheim, CA, USA

[11] C. Cranor, R. Ethington, A. Sehgal, D. Shur, C. Sreenan, J. E. Merwe,'Design and implementation of a distributed content management system," Proc. of the 13th intl. workshop on Net. \& oper. Sys. Sup. (NOSSDAV '03).ACM, USA, 4-11. DOI=10.1145/776322.776326.

[12] T. Plagemann,V. Goebel,A. Mauthe, L. Mathy, T. Turletti, G. Urvoy-Keller, "From Content Distribution Networks to Content Networks - Issues and Challenges, in Comp. Comm., Vol. 29(5), Mar 2006.

[13] C. Kim, Yu H. Bak, S. Woo, W. Lee, O. Min, H. Kim , "Design and implementation of a storage management method for content distribution," 8th Intl. Conf. Adv. 
Comm. Tech., ICACT'06, vol.2, pp.1147, Feb2006, doi: 10.1109/ICACT.2006.206173.

[14] P. Scheuermann, G. Weikum,P. Zabback,"Data partitioning and load balancing in parallel disk systems," VLDB Journal,vol. 7(1),pp.48-66,Feb 1998, DOI $=10.1007 / \mathrm{s} 007780050053$.

[15] Y. RynChoe, V. Pai, "Achieving Reliable Parallel Performance in a VoD Storage Server Using Randomization and Replication," IEEE Int. Par. \& Dist. Proc. Symp., IPDPS'07, Mar2007,doi: 10.1109/IPDPS.2007.370220.

[16] W. Tang,E. Wong,S. Chan,K. Ko, "Optimal video placement scheme for batching VOD services," IEEE Trans. on Broadcasting, vol.50(1), pp. 16- 25, Mar 2004,doi: 10.1109/TBC.2003.822983.

[17] K. Tang, K. Ko; S. Chan,E. Wong, "Optimal file placement in VOD system using genetic algorithm," IEEE Trans. on Ind. Elec., vol.48(5), pp.891-897, Oct 2001.

[18] Y. Wang, J. Liu, D. Du, J. Hsieh,"Efficient video file allocation schemes for video-on-demand services," Multimedia Syst. 5, pp.283-296,Sep.1997, DOI=10.1007/s005300050061.

[19] Y. Lin, H. Lai, Y. Lai , "A hierarchical network storage architecture for video-on-demand services," 21st Proc. IEEE Conf. on Loc. Com. Net.,1996,pp.355-364, Oct 1996 doi: 10.1109/LCN.1996.558164.

[20] V. Cholvi,J. Segarra, "Analysis and placement of storage capacity in large distributed video servers," Comput.Commun.pp.3604-3612, Sep. 2008, DOI=10.1016/j.comcom.2008.06.012.

[21] D. Brubeck,L. Rowe, "Hierarchical storage management in a distributed VOD system," Multimedia, IEEE, vol.3, no.3, pp.37-47, Fall 1996, doi: 10.1109/93.556538.

[22] S. Tsao, M. Chen, M. Ko, J. Ho, Y. Huang,"Data Allocation and Dynamic Load Balancing for Distributed Video Storage Server," Journal of Visual Comm.\& Image Rep., Vol.10(2),pp.197-218, June 1999, DOI: 10.1006/jvci.1999.0420.

[23] N.Laoutaris,V. Zissimopoulos,I. Stavrakakis," On the optimization of storage capacity allocation for content distribution," $\quad$ Comput.Netw., vol.47(3), Feb2005,pp.409-428,

DOI=10.1016/j.comnet.2004.07.020.

[24] I. Cidon,S. Kutten,R. Soffer,"Optimal allocation of electronic content," Proc of 20th Ann. Joint Conf. of IEEE Comp.r and Comm.Soc., INFOCOM'01,vol.3,pp.1773-1780,2001, doi:10.1109/INFCOM.2001.916675.
[25] C. Bisdikian, B. Patel,"Cost-based program allocation for distributed multimedia-on-demand systems," IEEE Multimedia,vol 3(3),pp.62-72,1996.

[26] K. Nwosu,P. Bobbie,B. Thuraisingham, "Data allocation and spatio-temporal implications for video-on-demand systems," Proc. Of 40th IEEE Ann. Int. Phoenix Conf. on Comp. \& Comm.,pp.629-635, Mar1995, doi: 10.1109/PCCC.1995.472427.

[27] K. Karlapalem,I. Ahmad, S. So,Y. Kwok,"Empirical evaluation of data allocation alg. for dist. multimedia database systems," Proc. Of 21th ann. Int. Comp. S।W \& App. Conf.,COMPSAC '97,pp.296-301,Aug1997 doi:10.1109/CMPSAC.1997.624842.

[28] J. Wang, R. Guha, "Efficiently Allocating Video Data in Distributed Multimedia Applications", Journal of App. Sys. Studies: Meth. and App. for Sys.Approaches, Special issue on Dist. Multimedia Sys. with App., November 2001.

[29] J. Kangasharju, J. Roberts, K. Ross, “Object Replication Strategies in Content Distribution Networks," Comp. Comm., vol. 25(3), pp. 367-383, March 2002.

[30] K. Tsang, S. Kwok, "Video Management in Commercial Distributed Video on Demand (VoD) Systems," PACIS 2000 Proceedings, paper 17.

[31] H. Ebara, Y. Abe, D. Ikeda, T. Tsutsui, K. Sakai, A. Nakaniwa, H. Okada, "A costeffectivedynamic content migration method in CDNs," IEICE Tran. On Comm. E88-B (12) (2005) 4598-4604.

[32] C. Xie, X. Li, Q. Wei,Q. Cao,” EOP: an efficient object placement and location algorithm for OBS cluster," Proc. of 7th int. conf. on Alg. and arch. for parallel processing (ICA3PP'07), Springer-Verlag, Berlin, Heidelberg, 222-230.

[33] D. Feng, Q. Lingjun, "Adaptive Object Placement in Object-Based Storage Systems with Minimal Blocking Probability," 20th Int. Conf. on Adv. Inf. Net. \& App.,AINA'06, 2006., vol.1, pp.611-616, April2006doi: 10.1109/AINA.2006.73

[34] Fati, S. M., Budiartu, R., \& Sumari, P. (2014, January). Provisioning virtual IPTV delivery networks using hybrid genetic algorithm. In Proceedings of the 8th International Conference on Ubiquitous Information Management and Communication (p. 106). ACM.

[35] Gaber, S. M. A., \& Sumari, P. (2012). Predictive and content-aware load balancing algorithm for peer-service area based IPTV networks. Multimedia Tools and Applications, 1-24. 